\title{
Serotonin Syndrome with Perioperative Oxycodone and Pregabalin
}

\section{TO THE EDITOR:}

Because various medications are administered perioperatively, physicians and anesthesiologists are aware of the possible interactions among pain control prescriptions. Pain control can benefit from co-administration of opioids and serotonergic agents. In the presence of serotonergic agents, oxycodone administration is often associated with the development of serotonin syndrome (SS) (1). Pregabalin (PGB) has been shown to be effective as an adjuvant therapy for postoperative pain (2) as well as neuropathic pain or anxiety disorder (3). This report is a description of the potential interaction between the combination of oxycodone/PGB and the risk of SS in the perioperative period.

A 75-year-old woman presented to the operating room for total knee replacement arthroplasty. One week earlier, she had undergone the same operation on her left knee joint. Her medical history was diabetes mellitus (HbA1c-NGSP: 6.0\%) for 20 years and hypertension. Her physical examination and routine laboratory studies were within normal limits. Her chest X-ray showed no active lung lesion. There was a right bundle branch block on electrocardiogram, and an echocardiogram revealed a frequent atrial premature beat and diastolic dysfunction grade 1 with left atrial enlargement with $62 \%$ of ejection fraction. The patient denied using illicit drugs and alcohol during the past year. The previous operation was performed under general inhalation anesthesia without a specific event.

On postoperative days (PODs) 0 and 1, postoperative pain was controlled using intravenous (IV) patient-controlled analgesia (PCA) containing fentanyl, ketorolac, and ramosetron (not available in the US) for preventing postoperative nausea and vomiting followed by daily oral PGB 150 mg, oxycodone hydrochloride $10 \mathrm{mg}$, and celecoxib $400 \mathrm{mg}$ with a divided dose. On POD 4, arterial hypoxemia occurred (pH 7.38; $\mathrm{PaO} 2$, 58 mm Hg; PaCO2, 47 mm Hg; HCO3, 27.8 mEq/L; SpO2, $89 \%$ ) with no pathologic changes in her chest X-ray, but this was resolved by supplying oxygen via a mask until the day assigned for the right-side total knee replacement arthroplasty.

Anesthesia was induced with propofol $150 \mathrm{mg}$ and rocuronium $50 \mathrm{mg}$ and maintained with desflurane (fraction of inspired oxygen $50 \%$ ) as required. The patient's hemodynamic profile remained stable throughout the 3 hour operation with mechanical ventilation. Lactated Ringer's solution $1.7 \mathrm{~L}$ was administered. The estimated blood loss was $350 \mathrm{~mL}$ and urine output was $300 \mathrm{~mL}$. The esophageal temperature was $36.2^{\circ} \mathrm{C}$ at the end of the operation. Neuromuscular blockade was reversed with IV pyridostigmine $10 \mathrm{mg}$ and glycopyrrolate $0.4 \mathrm{mg}$. The patient was extubated successfully, but showed delayed emergence even before the PCA loading dose. The extremities showed limited passive range of motion with rigidity and spontaneous clonus. Blood pressure and $\mathrm{SpO} 2$ level were in the normal range $(110 / 70,97 \%)$ with $6 \mathrm{~L} / \mathrm{min}$ via a mask, but the electrocardiogram showed sinus tachycardia (rate, 130/ min). Under close observation and IV hydration in the postanesthesia care unit, the muscle rigidity decreased slowly during the first hour, but the patient still showed lower extremity clonus and agitated behavior. While she awakened over time, she was confused and disoriented. Ankle clonus, agitation and cognitive dysfunction were resolved during her postanesthesia care unit stay. After transfer to the general ward, her postoperative course was uneventful and she was discharged with no neurologic sequelae.

SS results from serotonin hyper-stimulation at postsynaptic 5-hydroxytryptamine (HT)1A and possibly 5 -HT 2A receptors (4) by medications that increase serotonin levels. Hunter (5) described a triad of SS signs: an altered state of consciousness (confusion/disorientation/agitation), autonomic dysfunction (hypertension/ tachycardia/hyperthermia), and neuromuscular excitability (myoclonus/muscle rigidity/tremor). In addition to depressed mental status, thoracic muscle rigidity results in hypoventilation and hypoxemia. These signs warrant close observation because life-threatening conditions such as rhabdomyolysis, acute renal failure, and diffuse intravascular coagulation can occur even if clinical manifestations are relatively mild. SS is initiated within 24 hours of medication or dose changes, but it could occur spontaneously from a few days to weeks 
thereafter. Acquired vascular diseases such as hypertension, atherosclerosis, and hyperlipidemia are associated with a reduction in endothelial monoamine oxidase activity and reduced metabolism of serotonin (1).

Opioids do not directly stimulate serotonergic neuronal discharges but increase serotonin release in widespread areas of the forebrain (6). Fentanyl, a phenylpiperidine opioid, and ondansetron, a serotonin subtype 3 receptor antagonist, are also serotonin reuptake inhibitors that can provoke SS; however, there were no SS signs on POD 1 and 2 in the present case. Moreover, opioid-induced muscle rigidity could not be considered because PCA is unlikely to create a large bolus of fentanyl for administration. Oxycodone differs from most other opioids and co-administration of oxycodone with serotonin reuptake inhibitors is known to be associated with the development of SS (1).

Subchronic use of PGB $150 \mathrm{mg} / \mathrm{d}$ has little effect on cognitive or psychomotor function. However, in combination with oxycodone, patients taking PGB $150 \mathrm{mg}$ reported a heavy, sluggish feeling (7). In PGB intoxication, electroencephalography shows triphasic waves representing SS (8). It has been demonstrated that gabapentin, an analog of PGB, increases the whole-blood serotonin level when used at the clinical therapeutic dose range (9). There is the possibility of an increased serum concentration of PGB or serotonin level perioperatively in this case. Because metabolism of PGB in the liver is negligible, renal excretion is the primary route of clearance (3). Preoperative dehydration by withholding food and fluids by mouth, or reduced renal clearance impairment under long-standing hypertension may have contributed to a risk of PGB accumulation. Also, the risk of SS can be increased by peripheral serotonin metabolism impairment because of hypertension as a predisposing factor.

Appropriate treatments for SS are symptomatic support such as discontinuing suspicious agent(s), IV fluid administration, hypotensive/hypertensive therapy with vasoactive agents, and therapeutic hyperthermia with cooling. Neuromuscular blocking agents to minimize excessive muscle activity (clonus) and respiratory failure, renal failure, and coagulopathy should be managed. SS usually resolves within 24 hours of discontinuance of the suspicious agent(s) (10). Perioperative SS is described in a patient undergoing therapy with combinations of oxycodone and PGB to control postoperative pain. Some individuals are particularly at risk of augmentation of the serotonin level due to altered or impaired metabolism and should be monitored closely during the management of pain.

\section{Ho-Kyung, Song, MD.,PhD. \\ Professor}

Department of Anesthesiology and pain medicine, The Catholic University of Korea

Incheon St. Mary's Hospital

56 Dongsu-ro, Bupyong-gu

Incheon, S.Korea 403-720

Email : genovia@catholic.ac.kr

\section{References}

1. Karunatilake H, Buckley NA. Serotonin syndrome induced by fluvoxamine and oxycodone. Ann Pharmacother 2006; 40:155-157.

2. Sagit $M$, Yalcin S, Polat $H$, Korkmaz F, Cetinkaya S, Somdas MA. Efficacy of a single preoperative dose of pregabalin for postoperative pain after septoplasty. J Craniofac Surg 2013; 24:373-375.

3. Shneker BF, McAuley JW. Pregabalin: A new neuromodulator with broad therapeutic indications. Ann Pharmacother 2005; 39:2029-2037.

4. Gillman PK. A review of serotonin toxicity data: Implications for the mechanisms of antidepressant drug action. Biol Psychiatry 2006; 59:1046-1051.

5. Dunkley EJC, Isbister GK, Sibbritt D, Dawson AH, Whyte IM. The Hunter Serotonin Toxicity Criteria: Simple and accurate diagnostic decision rules for serotonin toxicity. QJM 2003; 96:635-642.

6. Codd EE, Shank RP, Schupsky JJ, Raffa RB. Serotonin and norepinephrine uptake inhibiting activity of centrally acting analgesics: Structural determinants and role in antinociception. J Pharm Exper Ther 1995; 274:1263-1270.

7. Zacny JP, Paice JA, Coalson DW. Subjective, psychomotor, and physiological ef- fects of pregabalin alone and in combination with oxycodone in healthy volunteers. Pharmacol Biochem Behav 2012; 100:560-565.

8. Lee S. Pregabalin intoxication-induced encephalopathy with triphasic waves. Epilepsy Behav 2012; 25:170-173.

9. Rao ML, Clarenbach P, Vahlensieck M, Krätzschmar S. Gabapentin augments whole blood serotonin in healthy young men. J Neural Transm 1988; 73:129-134.

10. Boyer EW, Shannon M. The serotonin syndrome. N Engl J Med 2005; 352:11121120. 